\title{
La cartera de servicios: diez años después
}

\author{
Para poder burlar los códigos \\ hay que entrar en los códigos
}

Roland Barthes

\begin{abstract}
$O$
esde hace 10 años la cartera de servicios se ha incorporado a nuestro entorno cotidiano. Muchas veces denostada y algunas veces defendida, pero ¿por qué nace? ¿para qué sirve? ¿tiene futuro?

La cartera de servicios nació hace 10 años y lo hizo respondiendo a una necesidad. A finales de la década de los 80 se produce, en todos los Estados de nuestro entorno socio-económico, la denominada crisis de bienestar y los sistemas sanitarios no se escapan al debate que se produce en todos los países. Se plantea una crisis que se engloba en tres apartados ${ }^{1}$ : la crisis financiera (incremento de los costes y de los gastos sanitarios por: extensión de la cobertura, incorporación de nueva tecnología, envejecimiento de la población, incremento de duración de la vida de personas gravemente incapacitadas, incremento de oferta de recursos, insuficiente aplicación de técnicas de organización y gestión en la prestación de la asistencia sanitaria,...etc.); la crisis de racionali dad (disminución de la eficacia y eficiencia de los sistemas sanitarios por: falta de coordinación con servicios sociales ante la mayor demanda de cuidados, la insuficiente atención a los aspectos preventivos y de promoción de la salud, el desarrollo de políticas intersectoriales y una mayor demanda de actividades de rehabilitación y reinserción social) y la crisis de legitimación (por una progresiva insatisfacción de los ciudadanos con su propia salud y con el sistema sanitario, que a su vez se relaciona con fenómenos como la comercialización de la salud, la medicalización de la vida cotidiana, la despersonalización de los individuos en el sistema sanitario, la gestión funcionarial de la solidaridad, la reducción del soporte social, el crecimiento del aparato burocrático para organizar la asistencia sanitaria. La "cosificación de la salud" deja en manos de la sanidad y de sus técnicos su manejo, convirtiendo a los ciudadanos en meros consumidores de los servicios sanitarios).

El impacto de estas crisis es distinto según el modelo de sistema sanitario, y nuestro sistema sanitario a pesar de alcanzar cotas aceptables de efectividad y eficiencia con el desarrollo del Sistema Nacional de Salud, no escapa de estas crisis. Ante esta situación se plantean algunas propuestas, entre las que cabe destacar las formuladas en $1991^{2}$, en las que se encuentran un núcleo razonable de reformas que cuentan con amplio soporte técnico. Éstas son:
\end{abstract}




\section{editorial}

-Necesidad de definición de un conjunto básico de prestaciones cubiertas por un Sistema Nacional de Salud consolidado: financiado públicamente, de aseguramiento universal y provisión pública de los servicios.

-Necesidad de separar las funciones de financiación, compra y pro visión. Este objetivo inspira la reforma realizada en 1992, de la estructura organizativa del Ministerio de Sanidad y Consumo, así como la Ordenación del Servicio Catalán de la Salud en 1990 y la propuesta del Sistema Vasco de Salud, aprobada por su parlamento en 1993.

- Aumentar la eficiencia de los servicios, dotando de mayor flexibilidad y autonomía en la gestión, así como introduciendo incentivos a la eficiencia basados en una competencia regulada.

- Promover mayores cuotas de participación social, libertad de elección y autorresponsabilidad en el cuidado y promoción de la salud y favorecer la actuación intersectorial.

La aceptación por el Ministerio de Sanidad de algunas de estas necesidades supuso el desarrollo de la planificación política o normativa (estimando necesidades de la salud y priorizando servicios y prestaciones: plan de salud); la planificación estratégica (aplicando los planes de salud a los servicios, reorientando servicios e introduciendo mejoras de gestión: los planes estratégicos de servicios) y la planificación táctica (estableciendo la cartera de servicios que respondiera a las necesidades identificadas, fijando la actividad de los servicios en sus dimensiones de efectividad, calidad y producción: Contrato Programa) ${ }^{2}$.

Según todos los planes de salud del estado, los problemas y necesidades de salud ${ }^{2,3}$ con mayor morbimortalidad en España son las enfermedades cardiovasculares, el cáncer, los accidentes, las enfermedades respiratorias, las enfermedades digestivas, los trastornos del aparato locomotor, las enfermedades transmisibles, el VIH-SIDA, la diabetes mellitus, la salud materno-infantil, la salud mental, la salud buco-dental y el envejecimiento. Si se asume que estas son las necesidades y problemas de salud más importantes, entenderemos que el Real Decreto 63/1995 de 20 de enero, de Prestaciones Sanitarias del Sistema Nacional de Salud de 1995 (cuyo objetivo final era proporcionar a los ciudadanos una garantía jurídica en sus relaciones con los servicios sanitarios y definir el alcance de la cobertura sanitaria), y consecuentemente las carteras de servicios, estén orientadas fundamentalmente a dichas áreas de necesidad.

En este marco en 1992-1993 se define por primera vez un plan de gestión que se plasma en un contrato-programa que tiene dos objetivos principales:

- Elaborar una estrategia revisable periódicamente y concretable anualmente, que vincule la política sanitaria con el funcionamiento operativo de los centros de salud.

- Informar a toda la organización sobre el análisis de la situación,

MEDIFAM Vol. 11 - Núm.10 - Diciembre 2001 
objetivos que se persiguen, medios disponibles para alcanzar dichos objetivos y las medidas de mejora que se pretenden introducir para conseguir los objetivos.

En este contexto está integrada la cartera de servicios.

La cartera de servicios es el catalogo de servicios que pretenden dar respuesta a los problemas de salud y a las necesidades prioritarias de nuestro país y cuya intervención puede y debe realizarse en AP. Los servicios incluidos en cartera representan el establecimiento y/o la consolidación de actividades fundamentadas científicamente para la consecución de los objetivos en salud, por lo que su seguimiento en cantidad y calidad es fundamental como medida de lo que el sistema sanitario puede aportar como variable a la consecución de la mejora en el nivel de salud. La línea seguida para su elaboración y actualización ha sido mediante técnicas de consenso basadas en criterios científico-técnicos, que buscan desde el inicio la implicación y participación de los profesionales de AP y de las sociedades científicas de AP.

La cartera de servicios pretendía responder a un triple objetivo.

1. Equilibrar los criterios institucionales con las demandas expresadas por los ciudadanos, respecto a los servicios que debería ofertar el sistema público a nuestro nivel.

2. Garantizar una oferta de servicios homogénea en todo el ámbito de la AP. Hasta ese momento, los servicios ofertados a los ciudadanos dependían en gran medida de los profesionales de cada centro de salud, de forma que la población recibía una mayor o menor cantidad de servicios en función del "voluntarismo" de los profesionales de su centro de referencia. Lo cual producía o podía inducir situaciones de clara desigualdad.

3. Servir como posible marco de referencia para crear modos de incentivación para aquellas unidades de trabajo que más y mejores servicios proporcionan a su población.

Pero la cartera de servicios no está exenta de críticas y estas están básicamente vinculadas a los siguientes aspectos: a) Recogen una parte muy limitada de la actividad cotidiana de los centros y algunos profesionales lo consideran una actividad marginal; b) en las definiciones de la cartera de servicios se establecen la actividad, los diagnósticos y los controles a realizar así como su periodicidad, pero no establecen los objetivos de esos controles ni se evalúa su consecución; c) son iguales para todos los EAP, y esto, que en inicio se alzaba como una garantía de equidad, provoca cierto alejamiento de los EAP con respecto a las necesidades y problemas de su zona básica de salud; d) no promueve mayores cuotas de participación social ni ha favorecido la actuación intersectorial; e) muchos profesionales lo sienten como algo extraño a ellos, como una herramienta centralizada e interna de la administración, y vivido por algunos con sentimiento de imposición; f) efectos perversos de la cartera de servicios en el sistema de incentivos. Ausencia de medidas correctoras sobre unidades no cumplidoras. Vinculación de cartera de 
servicios y sistemas de gestión de personal; y g) dificultades en su evaluación vinculadas a: evaluación del EAP versus evaluación de los profesionales; evaluación como fin en sí mismo: "registritis"; auditoría externa versus auditoría interna; evaluación más extensiva que intensiva; sesgos atribuibles a bases documentales; validez y fiabilidad; validez del producto medio; falta de sistema de registro homogéneo; evaluación compleja y rígida.

Con relación al primer punto, es cierto y debe hacerse un esfuerzo por definir todos los resultados que se esperan de un centro de salud, pero también es verdad que no debe olvidarse que, si queremos ser proactivos en nuestro sistema y mejorar en los niveles de salud, no debemos olvidar cuáles son las grandes áreas de necesidad y problemas que te nemos en nuestro país y, por lo tanto, seguir trabajando e insistiendo en esos aspectos. Existen cuatro propuestas sobre las que se tiene que seguir trabajando ${ }^{5}$, con una premisa previa y es que los objetivos de un sistema sanitario no son sólo la efectividad y la eficiencia, sino también la equidad y la viabilidad (en la que se encuentra la satisfacción de los usuarios, la motivación y los niveles de competencia de los profesiona les que hay que evaluar y potenciar su mejora).

Las cuatro pistas propuestas son:

1. Actualización de la oferta de servicios de los centros de salud y adaptación de la oferta de servicios a cada zona básica de salud.

Debe existir una oferta mínima común para todos los centros de salud, pero la variabilidad de las poblaciones exige un esfuerzo de adaptación de los servicios a estas y un esfuerzo claro hacia la intersectorialidad y la participación social.

2. Incorporación de la medición de los resultados de los procesos (resultados intermedios). Durante mucho tiempo, muchos autores han afirmado que los resultados (outcomes) rara vez son útiles para medir la calidad debido a la dificultad para recoger información válida sobre resultados clínicos por falta de información exacta, por estar presentes enfermedades concomitantes, por el largo periodo de tiempo para valorar los resultados y por la escasa potencia de algunos indicadores en poblaciones reducidas.

Y esto puede ser válido, probablemente, para los macroresultados sobre morbilidad y mortalidad, pero existen múltiples resultados claramente relacionados con el proceso (denominados resultados intermedios) y que sí parecen estar directamente ligados a la calidad de la atención. Actualmente a ningún médico de familia se nos escapa la importancia de conocer a nuestros pacientes diabéticos, saber su número y el número de veces que los vemos, o cuántas HbA1c les pedimos a lo largo del año, pero consideramos que probablemente nos gustaría más que se nos pidiera nuestro porcentaje de pacientes con control glicémico, perfil lipídico y tensional aceptables o, incluso, cuál es a lo largo del tiempo la evolución de lesiones en los pies o de ingresos hospitalarios. 
El sistema está maduro para estudiar la efectividad y eficiencia de los procesos cuya eficacia se haya demostrado con evidencias bien fun dadas.

Un elemento para su óptimo desarrollo es la adecuación de un sistema de información fiable, válido, sensible y especifico y sobre todo fácil.

3. Estandarización de los centros de salud.

Los resultados obtenidos en las evaluaciones de cartera de servicios de los distintos centros de salud no son comparables por razones sociodemográficas, por razones históricas de adscripción de recursos y por diferentes cargas de morbilidad. Pero, a pesar del tiempo, seguimos asistiendo a la comparación de resultados de la cartera de servicios entre centros de salud e incluso clasificando los centros y repartiendo incentivos en base a estos resultados. Esto es tan burdo como asumir comparaciones de tasas brutas sin estandarizar. Los resultados en salud pueden ser diferentes, no tanto por diferencias en la calidad sino por que los problemas de salud son de diferente importancia. En consecuencia, toda comparación de calidad asistencial exige una estandarización del con texto sociodemográfico, de la morbilidad inicial y de las cargas de trabajo, previa a la valoración de las diferencias observadas en los resultados. O se distribuyen equitativamente los recursos o se introducen medidas correctoras de sesgos.

4.- Medición de la productividad de los centros de salud ajustada a lo que se espera de ella y a la realidad de la población a la que atiende. De forma paralela a la mejora de la cartera de servicios se debe avanzar en la medición de la productividad de los centros de salud: ¿Cómo es nuestra eficacia de nivel, nuestra capacidad de resolución? ¿Cómo es nuestra gestión clínica (preventiva, diagnóstica y terapéutica)?

Para la medición de esta productividad existen diferentes métodos. En la actualidad existe múltiples iniciativas y entre ellas, el proyecto GESHIP de mejora de la AP, sobre la base de la medición del grado de resolución de los episodios tratados respecto a los esperados en base poblacional y epidemiológica, en relación con lo consumido e identificando niveles de calidad que condicionan esa relación. Su objetivo es la definición de los grados de productividad aplicando modelos de resolución de los episodios o agrupaciones de los mismos que se atienden con regularidad en $\mathrm{AP}^{7}$. Estos modelos deben considerarse complementarios al correcto desarrollo de la cartera de servicios en AP, por la sencilla razón que no debemos confundir la productividad con la efectividad.

La cartera de servicios cumplirá pronto diez años y ha hecho aportaciones importantes, pero es una herramienta dinámica y para que siga siendo operativa debe ser ampliada, adaptada y mejorada.

Verónica Casado Vicente

Presidente de la Sociedad Castellano-Leonesa de Medicina de Familia y Comunitaria 


\section{editorial}

\section{BIBLIOGRAFÍA}

1. Elola J. Crisis y reforma de la asistencia sanitaria pública en España (19831990) FIS. Madrid, 1991.

2. Elola J. Sistema Nacional de Salud: Evaluación de su eficiencia y alternativas de reforma. SG Editores. Barcelona 1994.

3. Casado V, Sevilla F, Elola J. El Plan de Salud del Ministerio de Sanidad y Consumo. Med Clin. 1998; 110: 265-74.

4. Plan de Salud. Madrid: Secretaría General Técnica. Centro de Publicaciones. Ministerio de Sanidad y Consumo, 1995; Nipo 351-95-02-7.

5. La cartera de servicios de Atención Primaria. Desarrollo estructural y metodológico. 1991-1999. Subdirección General de Atención Primaria. Area de Evaluación y Seguimiento. Insalud. Madrid. 2000.

6. Casado V. Opinión de la semFYC sobre la valoración de los resultados de la actividad de los centros de salud: Propuestas para evaluar resultados. M edifam. En prensa.

7. Ruiz Téllez A, Alonso López F. Sistemas de información maduros para una Atención Primaria adulta. El proyecto GESHIP. Medifam 2001; 11: 247-52.

\section{AClaración:}

En el editorial del Vol. $11 \mathrm{n}^{\circ} 9$ de la revista MEDIFAM, apareció como único firmante F. Borrell i Carrió, y debería haber aparecido también N. Cobb como coautor. 